\title{
Gallium Ga 68-labeled Affibody ABY-025
}

National Cancer Institute

\section{Source}

National Cancer Institute. Gallium Ga 68-labeled Affibody ABY-025. NCI Thesaurus. Code C107163.

A radioconjug ate composed of an optimized affibody conjug ated, via its C-terminal cysteine, to maleimide-DOTA, and linked to the radioisotope gallium Ga 68, with potential use as a tracer for human epidermal growth factor receptor type 2 (HER2; ErbB2)expressing tumors using positron emission tomography (PET). Upon intravenous administration of the gallium Ga 68-labeled affibody ABY-025, the affibody targets HER2expressing tumor cells. This facilitates both detection of HER2-expressing tumor cells and assessment of responses to HER2-targeting chemotherapeutic agents during PET imaging. The affibody is an optimized antibody mimetic based on a 6.5-kDa 3-helical bundle $Z$ domain derived from the staphylococcal protein A (Z her2:342); the nonbinding surface of the $Z$ domain is reeng ineered ( $Z$ her2:2891) to increase binding affinity for HER2 and to improve tumor imaging. HER2 is overexpressed in many cancer cell types. 